\title{
Validación empírica del CRAFFT Abuse Screening Test en una muestra de adolescentes españoles
}

\section{Empirical validation of the CRAFFT Abuse Screening Test in a Spanish sample}

\author{
Antonio Rial*, Sion Kim Harris**, John R.Knight**, Manuel Araujo*, Patricia Gómez*, \\ Teresa Braña*, Jesús Varela*, Sandra Golpe*. \\ * Universidad de Santiago de Compostela, España; ** Boston Children’s Hospital. Harvard Medical School.
}

\section{Resumen}

El CRAFFT Abuse Screening Test, desarrollado por el Center for Adolescents Substance Abuse Research (CeASAR) (Knight et al., 1999), es una herramienta de cribado del consumo de riesgo de alcohol y otras sustancias diseñada para su uso con adolescentes. Desde su publicación ha sido objeto de numerosas traducciones y validaciones en diferentes países, poblaciones y contextos que han dado cuenta de su enorme potencial. No obstante, seguimos sin disponer de estudios de validación empírica que garanticen su adecuado comportamiento psicométrico en España. El objetivo del presente trabajo consiste en desarrollar una versión adaptada del CRAFFT en castellano y analizar sus propiedades psicométricas en una muestra de adolescentes españoles. Para ello, se realizó una entrevista individual a 312 adolescentes de entre 12 y 18 años $(M=15,01 ; D T=1,83)$ de la comunidad gallega, que incluyó una parte de la Adolescent Diagnostic Interview (ADI) y del Problem Oriented Screening Instrument for Teenagers (POSIT). Los resultados obtenidos, similares a los encontrados en otros países, permiten informar que la versión española del CRAFFT presenta un buen comportamiento psicométrico. A nivel de consistencia interna se obtuvo un a de Cronbach satisfactorio de ,74. En cuanto a la sensibilidad y especificidad se obtuvieron unos valores del $74,4 \%$ y el $96,4 \%$ respectivamente, con un área bajo la curva COR de ,946. Por lo tanto, queda a disposición de investigadores y profesionales del ámbito de las conductas adictivas la versión española del CRAFFT, para que pueda ser utilizada en adelante con las garantías psicométricas necesarias.

Palabras clave: Adolescentes; Alcohol; CRAFFT; Cribado; Drogas.

\section{Abstract}

The CRAFFT Substance Abuse Screening Instrument, developed by the Center for Adolescents Substance Abuse Research (CeASAR) (Knight et al., 1999), is a screening tool for high-risk alcohol and drug risk consumption designed for use with adolescents. Since its publication it has been the subject of translations and validations in different countries, populations and contexts that have demonstrated its enormous potential. However, there is still no empirical validation study that would ensure its good psychometric performance in Spain. The aim of this paper is to develop an adapted version of the CRAFFT in Spanish and to analyze its psychometric properties in a sample of Spanish adolescents. For this purpose an individual interview was conducted on 312 adolescents aged between 12 and 18 years of age $(\mathrm{M}=15.01 ; \mathrm{SD}=1.83)$ from the Galician community. The interview included a part of the Adolescent Diagnostic Interview (ADI) and the Problem Oriented Screening Instrument for Teenagers (POSIT). The results obtained, similar to those found in other countries, allow us to report that the Spanish version of the CRAFFT has a good psychometric behaviorproperties. It was found to have a satisfactory internal consistency with a Cronbach's alpha value of .74. In terms of sensitivity and specificity, values of $74.4 \%$ and $96.4 \%$ respectively, were obtained and the area under the ROC curve was .946. The Spanish version of the CRAFFT is made available to researchers and professionals in the field of addictive behaviors, so that it can be used with the necessary psychometric guarantees.

Key words: Adolescents; Alcohol; CRAFFT; Screening; Drugs. 


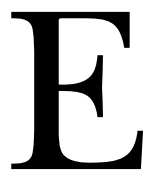

1 CRAFFT Substance Abuse Screening Test (Knight et al., 1999) es una de las herramientas más utilizadas para el screening del consumo de riesgo de alcohol y otras drogas en adolescentes (Mitchell et al., 2014). Tanto es así que su uso es recomendado por la guía "Alcohol Screening and Brief Intervention for Youth: Practicioner's Guide" (National Institute on Alcohol Abuse and Alcoholism [NIAAA], 2011) y por la American Academy of Pediatrics en su Policy Statement: "Substance use screening, brief intervention, and referral to treatment for pediatricians"(Committee on Substance Abuse, 2011).

Este instrumento de rápida y sencilla aplicación fue desarrollado en Boston, por el Center for Adolescent Substance Abuse Research (CeASAR) (Knight et al., 1999), con el objetivo de identificar de manera temprana a niños y jóvenes menores de 21 años que presentan un alto riesgo de desarrollar un trastorno por consumo de alcohol u otras drogas. Está constituido por 6 ítems de respuesta dicotómica (sí/no), precedidos de 3 ítems adicionales que actúan de filtro, que son puntuados con un 1 o un 0 en función de si el adolescente responde afirmativamente o no. La puntuación teórica de la escala oscila entre 0 y 12 , siendo 2 el punto de corte establecido por sus autores originales (Knight, et al., 1999) para hablar de un consumo de riesgo.

La necesidad de contar con instrumentos de detección en adolescentes se hace manifiesta, a la luz de los datos revelados por la última Encuesta Estatal sobre uso de Drogas en Enseñanzas Secundarias [ESTUDES 2014-2015] (Plan Nacional sobre Drogas, 2016). A pesar de que los niveles de consumo han disminuido en los últimos años, las cifras de prevalencia siguen siendo elevadas. Un 76,8\% de los estudiantes entre 14 y 18 años consumieron alcohol en el último año (68,2\% en el último mes); un $31,4 \%$ indicaron haber fumado tabaco en el último año (25,9\% en el último mes) y el $25,4 \%$ reconocieron haber consumido cannabis (18,6\% en el último mes). El resto de sustancias exploradas, tales como la cocaína, el éxtasis, las anfetaminas o los alucinógenos presentan cifras de prevalencia mucho menores, por debajo del $3 \%$.

A esos niveles de consumo, que a día de hoy todavía siguen siendo elevados, hay que añadir otras dos cuestiones que preocupan tanto a profesionales como investigadores. La primera de ellas tiene que ver con la temprana edad a la que los adolescentes se inician en el consumo de drogas. Así, por ejemplo, según datos del ESTUDES 2012-2013, la edad de inicio del consumo de alcohol se situaba en los 13,9 años, la del tabaco en 13,6 y la del cannabis en 14,9. A nivel europeo, el último informe del European School Survey Project on Alcohol and Other Drugs [ESPAD] (ESPAD Group, 2016) señala que un $47 \%$ de los estudiantes entre 16-18 años comenzó a consumir alcohol antes de los 14 años, el $23 \%$ tabaco y un $3 \%$ cannabis. La evidencia empírica disponible pone de manifiesto que la edad a la que los adolescentes se inician en el consumo de las diferentes sustancias no es algo banal (Cadaveira, 2009; Fontes et al., 2011). Por otra parte, desde hace unos años se viene observando un cambio en el patrón de consumo de alcohol entre los más jóvenes (Calafat y Juan, 2003; Sánchez, Moreno, Rivera y Ramos, 2015). La ingesta de grandes cantidades de alcohol en cortos espacios de tiempo, conocido como binge drinking o consumo intensivo de alcohol, constituye un grave problema sociosanitario, con claras consecuencias negativas (DeCamp, Gealt, Martin, O'Connell y Visher, 2015; López-Caneda et al., 2014; Moure et al., 2014; Parada et al., 2011).

La existencia de unas cifras de prevalencia de consumo de las diferentes sustancias (así como de binge drinking) todavía elevadas, unido a unas edades de inicio cada vez más tempranas (Cortés, Espejo y Giménez, 2007; Golpe, Isorna, Barreiro, Braña y Rial, 2017) no hace más que reforzar la necesidad de una detección precoz del consumo de alcohol y de otras drogas. Para ello resulta clave contar con herramientas de screening que además de estar adaptadas a nuestro país y contar con unas propiedades psicométricas contrastadas, sean breves, sencillas y fáciles de aplicar en una población cada vez más joven. En este sentido, el CRAFFT cuenta con algunas "bondades" que la convierten en una herramienta especialmente útil en este contexto. En primer lugar, se trata de un instrumento muy fácil de aplicar, habida cuenta el reducido número de ítems que lo componen. En segundo lugar, cabe destacar que cuenta con un alto grado de implantación a nivel internacional y una amplia tradición de estudios de validación en diferentes países, contextos y poblaciones, que dan cuenta de su adecuado comportamiento psicométrico. Y, por último, es un instrumento que tiene una vinculación directa con los planes y estrategias existentes a nivel de prevención, en la medida en que ha sido implantado en programas de detección precoz e intervención breve (SBIRT, Screening, Brief Intervention and Referral to Treatment) (Committee on Substance Abuse, 2011; Harris, Louis-Jacques y Knight, 2014; Pilowsky y Wu, 2013).

Desde su publicación el CRAFFT ha sido objeto de numerosas traducciones y validaciones en diferentes países, poblaciones y contextos. A nivel de consistencia interna, la revisión de numerosos trabajos revela unos valores más bien discretos con índices $\alpha$ de Cronbach que en algunos casos no llegan al ,70 (Bertini et al., 2015; Knight, Sherritt, Shrier, Harris y Chang, 2002; Skogen, BØe, Knudsen y Hysing, 2013; Wartberg, Kriston, Diestelkamp, Arnaud y Thomasius, 2016). En cuanto a su capacidad de cribado, es posible señalar que el CRAFFT posee unas propiedades psicométricas adecuadas (Dhalla, Zumbo y Poole, 2011) con unos índices de sensibilidad y especificidad, por lo general, elevados (Gryczynski et al., 2015; Kandemir et al., 2015; Knight, Sherritt, Harris, Gates y Chang, 2003; Pereira, Schram y Azevedo, 2016). No obstante, en aquellos trabajos en los que se incluyen además los valores predictivos, 
por lo general alguno de los cuatro indicadores presenta un resultado más pobre (Cook, Chung, Kelly y Clark, 2005; Kelly, Donovan, Chung, Cook y Delbridge, 2004; Knight et al., 1999). En cualquier caso es importante señalar que las diferencias metodológicas entre los diferentes estudios, como por ejemplo la utilización de diferentes criterios estándar, producen cierta incertidumbre en la interpretación de dichas propiedades. Respecto a la estructura factorial del CRAFFT diferentes trabajos apoyan la unidimensionalidad de la escala (Subramaniam, Cheok, Verma, Wong y Chong, 2010; Wartberg et al., 2016).

En cuanto a las versiones existentes de la escala en el idioma español, se pueden encontrar hasta tres versiones diferentes. Por una parte, existe una traducción al español que los propios autores originales del CRAFFT ofrecen en su página web, y por otra, existen dos versiones distintas adaptadas a Colombia (Cote-Menendez, Uribe-Isaza y Prieto-Suárez, 2013; Pérez y Scoppetta, 2011). No obstante, la redacción de los ítems en cualquiera de las tres versiones se encuentra adaptada al idioma español en Latinoamérica por lo que no serían aplicables al contexto de España. De este modo, a día de hoy seguimos sin disponer de una versión del CRAFFT debidamente adaptada y validada en España, que permita que profesionales e investigadores puedan hacer uso de él con garantías.

El objetivo del presente estudio consiste precisamente en realizar una adaptación al español del CRAFFT Abuse Screening Test y analizar su comportamiento psicométrico en una muestra de adolescentes españoles. De manera más concreta, la hipótesis que se intentará poner a prueba en el estudio empírico es que la versión española del CRAFFT constituye un instrumento adecuado a nivel psicométrico para detectar precozmente problemas de consumo de alcohol y/u otras sustancias en adolescentes españoles.

\section{Método}

\section{Participantes}

Para dar cuenta del objetivo señalado se recurrió a una metodología selectiva consistente en la realización de entrevistas individuales a estudiantes de Educación Secundaria Obligatoria (ESO), Bachillerato y Ciclos Formativos de grado medio de la comunidad autónoma de Galicia (España). Para la selección de la muestra se recurrió a un muestreo bietápico: por conglomerados, para la selección de las unidades de primer nivel (centros educativos) y por cuotas, para la selección de las unidades de segundo nivel (individuos).

A pesar de que inicialmente fueron entrevistados un total de 343 adolescentes, la muestra final estuvo compuesta por 312 individuos, descartándose 31 fundamentalmente por no haber podido completar la entrevista en su totalidad o por incoherencias manifiestas en las respuestas. Para garantizar que no existía ningún sesgo en la distribución de los casos missingy que éstos se distribuían de forma alea- toria, se comprobó que el porcentaje de casos perdidos fuese similar en los diferentes segmentos muestrales en función del género, grupo de edad, titularidad del centro y entorno de residencia, calculando para ello estadísticos de contraste $\chi^{2}$.

Respecto a la composición de la muestra, el 56,4\% eran hombres y el 43,6 \% mujeres y sus edades estaban comprendidas entre los 12 y los 18 años $(M=15,01 ; D T=1,83)$. Los participantes fueron seleccionados aleatoriamente de un total de 33 centros educativos (22 públicos y 11 privados). De estos, el 64,9\% se encontraban cursando la ESO (32,6\% en el primer ciclo y el $32,3 \%$ en el segundo), el 21,3\% en Bachillerato y el 13,9\% FP Básica o un Ciclo Formativo de grado medio. Por último, el 42,4\% residían en un entorno urbano y el $57,6 \%$ rural o semirural.

\section{Instrumento}

Los datos fueron recogidos mediante una entrevista estructurada, con el apoyo de un cuestionario en el que se incluían: el CRAFFT Abuse Screening Test (Knight et al., 1999), la Adolescent Diagnostic Interview (ADI) (Winters y Henly, 1993) y la subescala de Uso y Abuso de Sustancias del Problem Oriented Screening Instrument for Teenagers (POSIT $_{\text {UAS }}$ ) (Rahdert, 1991). Para evitar un posible sesgo del orden de cumplimentación de los tres instrumentos, éste fue debidamente contrabalanceado.

El CRAFFT (Knight, et al., 1999) es una herramienta compuesta de tan solo 6 ítems dicotómicos (sí/no), diseñada específicamente para el screening del consumo de riesgo de alcohol y otras sustancias en adolescentes. La administración del CRAFFT comienza con 3 preguntas de apertura. Si el adolescente contesta "no" a esas preguntas, el entrevistador únicamente necesitará preguntarle la primera cuestión del CRAFFT. Si el adolescente contesta "sí" a una o más de las 3 preguntas de apertura, el entrevistador preguntará los 6 ítems que forman el CRAFFT propiamente dicho. Para su empleo en este trabajo el CRAFFT fue debidamente traducido y contra-traducido bajo la supervisión de sus autores originales. Una vez adaptado al castellano, se llevó a cabo un estudio piloto con el objetivo de evaluar el nivel de comprensión de las diferentes preguntas y la claridad de las mismas. La muestra estuvo compuesta por 51 adolescentes de entre 12 y 17 años $(M=14,36 ; D T=1,47)$. Para la selección de la muestra se utilizó un muestreo accidental, si bien se intentó que los participantes abarcasen el rango de edad del target de interés. Los datos fueron recogidos en 5 localidades diferentes, intentado con ello contar con sujetos de entornos diferentes (urbano, rural y semirural). Para la recogida de datos se diseñó un cuestionario, administrado a través de una entrevista personal, con tres bloques diferenciados. En el primer bloque se incluía el CRAFFT; en el segundo, se incluyeron 4 preguntas de una entrevista cognitiva (Paradigma Probing Based - Procedimiento Delayed Retrospective Probing) encaminadas a conocer el 
grado de comprensión del mismo y, por último, se incluyó un breve apartado de carácter sociodemográfico (género, edad y curso). Los resultados del estudio piloto indicaron que el CRAFFT es una herramienta realmente breve, clara y de fácil comprensión.

La Adolescent Diagnostic Interview (ADI) (Winters y Henly, 1993) fue utilizada como criterio para calcular la sensibilidad, especificidad, el valor predictivo positivo (VPP) y negativo (VPN) del CRAFFT y consiste en una entrevista diagnóstica de 213 ítems adaptada a los criterios del DSM5 (American Psychiatric Association [APA], 2013), para la identificación de trastornos por consumo de sustancias en adolescentes. Para su empleo en este trabajo, dicha entrevista fue traducida y contra-traducida bajo la supervisión de sus autores originales. La fiabilidad de las distintas escalas diagnósticas fue elevada obteniendo un valor $\alpha$ de Cronbach de ,88 para el diagnóstico de un trastorno por consumo de alcohol; de ,89 para el diagnóstico de un trastorno por consumo de cannabis y de ,92 para el diagnóstico de un trastorno por consumo de sustancias, siendo estos valores muy similares a los obtenidos en el trabajo de Araujo, Golpe, Braña y Varela (2018).

Por último, como indicador complementario de validez de criterio se incluyó la subescala de Uso y Abuso de Sustancias del Problem Oriented Screening Instrument for Teenagers $\left(\right.$ POSIT $_{\text {UAS }}$ ) (Rahdert, 1991), que consta de 17 ítems dicotómicos (sí/no) y que fue validado en el trabajo de Araujo et al. (2018), presentando una buena fiabilidad ( $\alpha$ de Cronbach de ,82), al igual que unos elevados valores de sensibilidad $(94,3 \%)$ y especificidad $(83,9 \%)$.

\section{Procedimiento}

Los datos fueron recogidos a través de una entrevista personal realizada en los propios centros educativos, en despachos habilitados para tal fin, por un equipo de psicólogos/as con experiencia en este tipo de trabajos. La duración de cada entrevista osciló entre 45 y 60 minutos. Los participantes fueron informados de la finalidad del estudio, así como del anonimato y la confidencialidad de sus respuestas. El estudio contó con el consentimiento y colaboración tanto de la dirección de los centros como de las respectivas asociaciones de padres/madres. La participación fue totalmente voluntaria y no remunerada. El estudio fue aprobado por el Comité Bioético de la Universidad de Santiago de Compostela.

\section{Análisis de datos}

En primer lugar se realizó un análisis descriptivo mediante el cálculo de porcentajes, así como de los estadísticos de tendencia central y de dispersión. Además se realizaron comparaciones de medias en función del género (mediante la aplicación de la prueba $t$ de Student) y del grupo de edad (a través de un Anova unifactorial y un contraste post-hoc de Tukey). Dado el incumplimiento de la normalidad se aplicó de manera complementaria la prueba U de Mann-Whitney para 2 grupos y la prueba de Kruskal-Wallis para más de 2 grupos. Para evaluar la consistencia interna se calculó el índice KR-20, adecuado para variables dicotómicas (Kuder y Richardson, 1937) y el Coeficiente Omega $(\Omega)$. Para analizar las propiedades psicométricas de la escala se calcularon los índices de sensibilidad, especificidad, VPP y VPN. De manera complementaria, se calculó el área bajo la curva ROC (Receiver's Operating Characteristics) con el objetivo de determinar el punto de corte óptimo. Por último, para evaluar la validez de criterio se analizó el grado de concordancia del CRAFFT con el POSIT ${ }_{\text {UAS }}$ Los análisis fueron realizados con el paquete estadístico IBM SPSS Statistics 20.

\section{Resultados}

\section{Descriptivos}

En la Tabla 1 se presentan las respuestas directas de los 312 adolescentes a cada uno de los 9 ítems que componen el CRAFFT, con el porcentaje de sujetos que respondieron afirmativamente a cada uno de ellos. Como se puede

Tabla 1. Porcentaje de respuestas afirmativas a cada uno de los ítems del CRAFFT

\begin{tabular}{|c|c|}
\hline Ítem & \% sí \\
\hline En los últimos 12 meses ¿has consumido bebidas alcohólicas (más de unos pocos tragos)? & 47,8 \\
\hline En los últimos 12 meses ¿has fumado marihuana o hachís? & 18,3 \\
\hline $\begin{array}{l}\text { En los últimos } 12 \text { meses ¿has tomado alguna otra sustancia para "colocarte” (drogas ilegales, pastillas, medicamentos o alguna sustancia } \\
\text { esnifada o inhalada)? }\end{array}$ & 4,2 \\
\hline $\begin{array}{l}\text { ¿Alguna vez has ido en un coche/moto conducido por alguien (incluido tú mismo) que hubiese bebido alcohol o consumido algún tipo de } \\
\text { droga? }\end{array}$ & 25,7 \\
\hline ¿Alguna vez has consumido alcohol o algún tipo de droga para relajarte, sentirte mejor contigo mismo o encajar en un grupo? & 29,7 \\
\hline ¿Alguna vez has consumido alcohol o algún tipo de droga estando tú solo/a, sin compañía? & 32,9 \\
\hline ¿Alguna vez te has olvidado de cosas que hiciste mientras consumías alcohol o algún tipo de droga? & 45,2 \\
\hline ¿Alguna vez tu familia o amigos te han dicho que deberías reducir tu consumo de alcohol o drogas? & 27,7 \\
\hline ¿Alguna vez te has metido en líos mientras consumías alcohol o algún tipo de droga? & 22,7 \\
\hline
\end{tabular}


observar, casi el $50 \%$ de los adolescentes señalaron haber consumido alcohol en el último año, el 18,3\% marihuana o hachís y el 4,2\% alguna otra sustancia. Si atendemos a los ítems que conforman el CRAFFT propiamente dicho (del 4 al 9), el mayor porcentaje se corresponde con el ítem 7 (“¿alguna vez te has olvidado de cosas que hiciste mientras consumías alcohol o algún tipo de droga?”) con un 45,2\% de respuestas afirmativas. Por su parte, el ítem 9 (“¿alguna vez te has metido en líos mientras consumías alcohol o algún tipo de droga?") es el que presenta un menor porcentaje de respuestas afirmativas $(22,7 \%)$.

Los estadísticos descriptivos para la puntuación total se recogen en la Tabla 2. La media global del CRAFFT es 1,05 y la desviación típica 1,60, con un rango de puntuaciones observado entre 0 y 6 . Los estadísticos de asimetría y curtosis estandarizados revelan la existencia de asimetría positiva y una distribución leptocúrtica, lo cual pone de manifiesto que las puntuaciones no siguen una distribución normal. El incumplimiento de la normalidad fue contrastado mediante la prueba de Kolmogorov-Smirnov, con la correspondiente corrección de Lilliefors ( $\mathrm{K}-\mathrm{S}=0,302 ; p<, 001)$.

Tabla 2. Descriptivos para la puntuación total del CRAFFT

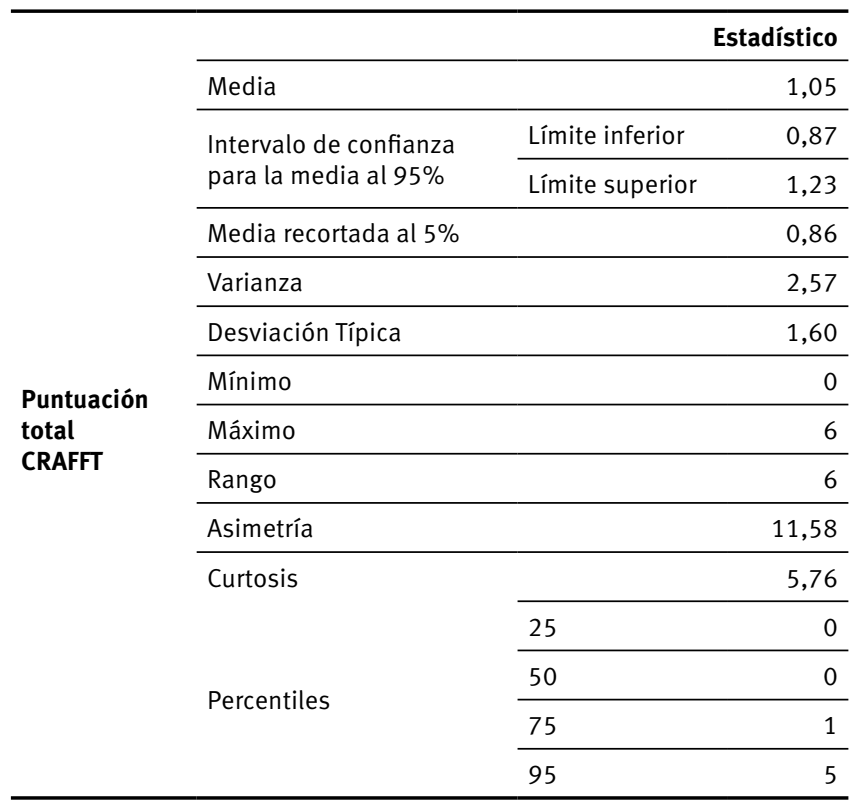

A continuación se recoge la distribución de frecuencias y los porcentajes acumulados para las diferentes puntuaciones. Adoptando el punto de corte original de la escala $(\geq 2)$ es posible señalar que un $22,9 \%$ de la muestra presenta un resultado positivo en el CRAFFT.

Cuando se comparan las puntuaciones medias por sexo, se observa que si bien las mujeres obtienen una puntuación inferior a la de los hombres $(0,94$ vs 1,13$)$ esta diferencia no llega a ser estadísticamente significativa $(t=1,07$; $p=, 29 ; Z=-, 09 ; p=, 93)$. Por lo que se refiere a la edad, las diferencias entre los tres grupos establecidos (12-14 años, 15-16 y 17-18) sí son estadísticamente significativas $\left(\mathrm{F}=50,567 ; p<, 001 ; \chi^{2}=84,87 ; p<, 001\right)$, siendo el grupo de 17-18 años el que presenta un promedio mayor $(2,20)$, seguido del de 15-16 $(1,04)$ y el de $12-14(0,23)$.

\section{Fiabilidad de las puntuaciones}

Como evidencia de la fiabilidad del CRAFFT se calculó su consistencia interna. Ésta fue evaluada mediante el cálculo del índice KR-20, resultando aceptable, con un valor a de ,74. Además, se calculó el coeficiente Omega, obteniendo un valor $\Omega$ de , 82 .

Se analizó también la consistencia de cada ítem de forma individual, a través del cálculo del Índice de Homogeneidad Corregido (IHC), obteniendo valores entre ,38 y ,61. Los ítems 2 y 4 fueron los que se mostraron menos consistentes con el conjunto de la escala. Sin embargo, la eliminación de ninguno de ellos reportaba mejora alguna sobre la consistencia global de la escala (Tabla 4).

Tabla 4. Consistencia de los ítems del CRAFFT

\begin{tabular}{lcc}
\hline Item & $\begin{array}{c}\text { KR-20 } \\
\text { si se elimina el ítem }\end{array}$ & IHC \\
\hline 1 &, 714 &, 431 \\
2 &, 726 &, 385 \\
3 &, 697 &, 487 \\
4 &, 729 &, 384 \\
5 &, 671 &, 583 \\
6 &, 668 &, 605 \\
\hline GLOBAL & & \\
\hline
\end{tabular}

Tabla 3. Distribución de frecuencias para la puntuación total del CRAFFT

\begin{tabular}{cccc}
\hline Puntuación Total & Frecuencia & Porcentaje válido & Porcentaje acumulado \\
\hline 0 & 173 & $55,8 \%$ & $55,8 \%$ \\
1 & 65 & $21,3 \%$ & $77,1 \%$ \\
2 & 17 & $5,5 \%$ & $82,6 \%$ \\
3 & 19 & $6,1 \%$ & $88,7 \%$ \\
4 & 14 & $4,5 \%$ & $93,2 \%$ \\
5 & 14 & $4,5 \%$ & $97,7 \%$ \\
6 & 7 & $2,3 \%$ & $100 \%$ \\
\hline
\end{tabular}




\section{Sensibilidad, especificidad, VPP y VPN y Curva ROC}

Los valores de sensibilidad, especificidad, VPP y VPN para distintos puntos de corte se recogen en la tabla 5 . Los resultados obtenidos permiten señalar que el CRAFFT muestra un buen comportamiento psicométrico, tanto si se adopta el punto de corte 1 como el 2, siendo este último el que alcanza un mayor equilibrio entre los cuatro indicadores.

Cuando se utiliza el punto de corte original $(\geq 2)$ el CRAFFT alcanza una sensibilidad del $74,4 \%$ y una especificidad del 96,4\%. En otras palabras, es capaz de detectar verdaderos positivos en el 74,4\% de los casos y de rechazar verdaderos negativos en el 96,4\%, siendo ambos resultados muy aceptables. Si nos fijamos en los valores predictivos obtenidos para este punto de corte observamos que el valor predictivo positivo es del $88,4 \%$, mientras que el valor predictivo negativo es del 91,1\%. Esto quiere decir que la probabilidad de que un adolescente que ha obtenido un resultado positivo en el CRAFFT presente realmente un trastorno por consumo de sustancias es del $88,4 \%$ y la probabilidad de que un adolescente que ha dado negativo no presente ningún trastorno es del 91,1\%. Por su parte, cuando se rebaja el punto de corte a 1 , lo que se consigue es maximizar el índice de sensibilidad $(97,6 \%)$ y del valor predictivo negativo $(98,8 \%)$ en detrimento de la especificidad $(76,6 \%)$ y el valor predictivo positivo $(60,6 \%)$. De manera complementaria, se llevó a cabo un Análisis de Curva ROC (Receiver Operating Characteristic), alcanzando un área bajo la curva de ,946 (Figura 1).

Si adoptamos el punto de corte original y analizamos las propiedades psicométricas del CRAFFT atendiendo al género, se puede observar el que el CRAFFT presenta un mejor comportamiento en el caso de los hombres que de las mujeres. En cuanto a la edad, los resultados son aceptables en los tres grupos considerados, especialmente en el de $12-14$ años (sensibilidad $=75 \%$; especificidad $=100 \%$; $\mathrm{VPP}=100 \%$ y $\mathrm{VPN}=99,1 \%$ ), empeorando ligeramente todos los índices a medida que aumenta la edad.

\section{Evidencias de validez de relación con variables externas}

Para estudiar la validez de criterio se comparó, en primer lugar, el porcentaje de adolescentes que dieron positivo en el CRAFFT y en el ADI (22,9\% y 26,8\%, respectivamente), obteniéndose un índice Kappa de concordancia de ,75 $(p<, 001)$. En segundo lugar, se realizó esa misma comparación entre el CRAFFT y el POSIT $_{\text {UAS }}$, siendo el porcentaje de positivos en esta última herramienta del $39,4 \%$, con un índice de concordancia Kappa de ,67 $(p<$ ,001). Por último, se calculó también el coeficiente de correlación de Pearson entre las puntuaciones del CRAFFT

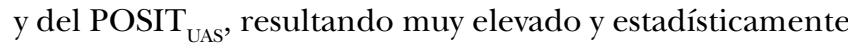
significativo $\left(\mathrm{r}_{\mathrm{xy}}=, 86 ; p<, 001\right)$.

\section{Evidencias de validez de estructura interna}

Se dividió la muestra en dos mitades de manera aleatoria. Con la primera de ellas se ejecutó un Análisis Fac-

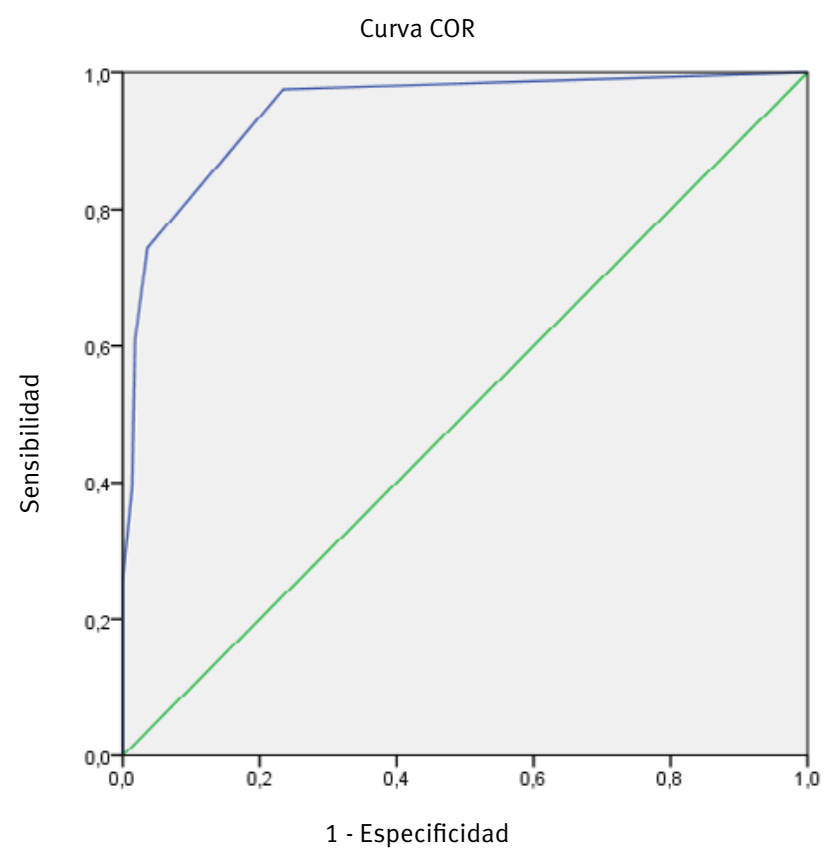

Figura 1. Curva COR del CRAFFT

Tabla 5. Propiedades psicométricas del CRAFFT

\begin{tabular}{|c|c|c|c|c|c|c|}
\hline & & Sensibilidad (\%) & Especificidad (\%) & VPP (\%) & VPN (\%) & CURVA ROC \\
\hline Corte $\geq 1$ & & 97,6 & 76,6 & 60,6 & 98,8 & \\
\hline Corte $\geq 2$ & & 74,4 & 96,4 & 88,4 & 91,1 & ,946 \\
\hline \multirow[t]{3}{*}{ Corte $\geq 3$} & & 61 & 98,2 & 92,6 & 87,2 & \\
\hline & & Sensibilidad (\%) & Especificidad (\%) & VPP (\%) & VPN (\%) & CURVA ROC \\
\hline & & & & Corte $\geq 2$ & & \\
\hline \multirow{2}{*}{ Género } & Hombres & 76,9 & 97,5 & 93 & 90,8 & ,958 \\
\hline & Mujeres & 70 & 95 & 80,8 & 91,4 & ,926 \\
\hline \multirow{3}{*}{ Edad } & 12-14 años & 75 & 100 & 100 & 99,1 & ,984 \\
\hline & 15-16 años & 74,1 & 97,2 & 90,9 & 90,9 & ,913 \\
\hline & 17-18 años & 74,5 & 82,9 & 86,4 & 69 & ,892 \\
\hline
\end{tabular}


torial Exploratorio (AFE) y con la segunda un Análisis Factorial Confirmatorio (AFC). Para la realización del AFE se utilizó como método de extracción de factores el Método de Componentes Principales. El índice KMO fue de ,77 y el Test de Esfericidad de Barlett de 184,61 $(p<$ 0,001). El análisis proporcionó 1 factor, que explicaba el $44,30 \%$ de la varianza de los datos. Con la segunda mitad de la muestra se llevó a cabo un AFC, con el objetivo de confirmar esta estructura unidimensional. Las saturaciones factoriales estandarizadas fueron superiores a , 45 y los índices de bondad de ajuste obtenidos (GFI, Goodness of Fit Index; AGFI, Adjusted Goodness of Fit Index; NFI, Normed Fit Index y RMR, Root Mean Square Residual), presentaron valores muy aceptables (GFI = ,995; AGFI = ,989 y NFI = ,990) acordes a los criterios recogidos por Byrne (2009) y Kline (2005).

\section{Discusión}

Uno de los instrumentos para el screening del consumo de riesgo de drogas que tiene mayor implantación a nivel mundial es, sin duda, el CRAFFT. Su utilización en diferentes países y en distintos contextos ha dado muestras de su enorme potencial (Agley, Gassman, Jun, Nowicke y Samuel, 2015; Bernard et al., 2005; Cote-Menendez et al., 2013; Cummins et al., 2003; Dieppe, Stanhope y Rakhra, 2009; Harris et al., 2016, 2014; Karila et al., 2007; Van Weelden et al., 2016). En España, aunque algunos profesionales e investigadores han utilizado ocasionalmente el CRAFFT, todavía no existen estudios psicométricos que garanticen el adecuado funcionamiento de este instrumento en nuestro país.

Los resultados obtenidos a partir de una muestra de 312 estudiantes de la comunidad gallega permiten constatar que el CRAFFT tiene un buen comportamiento psicométrico. En primer lugar, a nivel de consistencia interna, se ha obtenido un valor $\alpha$ satisfactorio de ,74, superior al obtenido en el estudio de validación original de Knight et al. (2002) $(\alpha=, 68)$ y al de otros trabajos (Bertini et al., 2015; Kelly et al., 2004; Subramaniam et al., 2010; Wartberg et al., 2016). En segundo lugar, a nivel de cribado, el punto de corte original $(\geq 2)$ es el que presenta un mayor equilibrio entre los cuatro indicadores utilizados, con una sensibilidad del $74,4 \%$, una especificidad del $96,4 \%$, un VPP del $88,4 \%$ y un VPN del $91,1 \%$. No obstante, si atendemos a la recomendación realizada por Latimer, Winters y Stinchfield (1997), según la cual ante una herramienta de screening lo más importante es maximizar la sensibilidad para evitar que un adolescente con abuso de drogas sea omitido en el screening, convendría replantearse la posibilidad de rebajar el punto de corte a 1, tal y como ya hicieran Subramaniam et al. (2010) o Skogen et al. (2013). Los resultados obtenidos por segmentos, utilizando el punto de corte original, ponen de manifiesto que el CRAFFT presenta un buen comportamiento psicométrico tanto en chicos como en chicas, al igual que en los diferentes grupos de edad. Sin embargo, conviene señalar que las chicas obtienen unos valores en los cuatro indicadores ligeramente inferiores al de los chicos, y lo mismo ocurre a medida que aumenta la edad.

En cuanto a la validez de constructo del CRAFFT, los análisis realizados han permitido confirmar la estructura unidimensional de la escala, tal y como ya habían señalado los trabajos de Subramaniam et al. (2010) y Wartberg et al. (2016).

Por último, los elevados índices de concordancia del

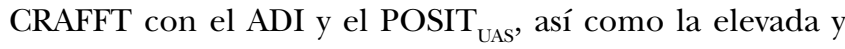
significativa correlación hallada entre el CRAFFT y el POSIT $_{\text {UAS }}$ dan cuenta de su validez de criterio.

En definitiva, el presente trabajo permite poner a disposición de investigadores y profesionales del ámbito de las conductas adictivas una versión adaptada y empíricamente validada del CRAFFT Abuse Screening Test. Los resultados obtenidos ponen de manifiesto que el CRAFFT presenta un buen comportamiento psicométrico y constituye una herramienta apropiada para ser utilizada dentro del contexto escolar. Además se ha constatado que el hecho de ser administrada por personal no sanitario no implica merma alguna de sus propiedades, lo cual amplía su potencial y sus posibilidades de utilización. Asimismo, este trabajo abre la posibilidad a que el CRAFFT pueda ser utilizado como herramienta de cribado en el marco de posibles programas de detección precoz e intervención breve (SBIRT) a desarrollar en nuestro país.

No obstante, es importante señalar algunas limitaciones de este estudio. Desde el punto de vista muestral cabe destacar que aunque el tamaño de la muestra (312 adolescentes) sea similar o incluso superior al de otros estudios de validación (Bernard et al., 2005; Bertini et al., 2015; Cummins et al., 2003; Kelly et al., 2004) resulta insuficiente para realizar una baremación del instrumento atendiendo a diferentes segmentos sociodemográficos. Por otra parte, el hecho de que sólo se hayan utilizado adolescentes de la comunidad autónoma de Galicia constituye en sí mismo un condicionante de la validez externa. Para intentar atenuar dicha limitación, se han incluido en la muestra estudiantes tanto de centros públicos como privados y concertados, residentes tanto en entornos urbanos, como rurales o semirurales. No obstante, es obvio que futuros trabajos han de marcarse como objetivo analizar el comportamiento psicométrico de la escala en otras comunidades.

Hubiese sido de interés también poder disponer de información muestral acerca de algunos aspectos clínicos, como la presencia de diagnóstico comórbido, la existencia de historia familiar de trastorno, etc. Sin embargo, conviene advertir que se trata de un primer estudio de validación en el ámbito escolar, contexto en el que pretende ser utilizado el instrumento de forma más inmediata. 
Por último, el hecho de que los datos hayan sido recogidos en el contexto escolar y no en los servicios de Atención Primaria mediante una entrevista clínica como tal, hace que las variables analizadas hayan sido autoinformadas, por lo que es imposible conocer objetivamente en qué medida los adolescentes pueden realmente haber infraestimado o sobreestimado sus niveles de consumo. No obstante, como han señalado previamente diferentes expertos del ámbito de las conductas adictivas, como los propios Babor, De La Fuente, Saunders y Grant (1989) o Winters, Stinchfield, Henly y Schwartz (1990), las medidas de autoinforme han demostrado ser fiables e incluso mejores que otros métodos a la hora de evaluar los niveles de consumo de alcohol y otras drogas. Por otra parte, el hecho de validar el CRAFFT en el contexto escolar lo convierte en una herramienta de enorme potencial aplicado, habida cuenta de que es precisamente en este ámbito donde se viene desarrollando en nuestro país buena parte del trabajo preventivo.

\section{Reconocimientos}

Los autores de este trabajo quieren agradecer la financiación recibida a través de la Delegación del Gobierno para el Plan Nacional sobre Drogas (Ref. 2013/046) para la realización de este estudio.

\section{Conflicto de intereses}

Los autores de este artículo declaran no tener ningún conflicto de intereses

\section{Referencias}

Agley, J., Gassman, R. A., Jun, M., Nowicke, C. y Samuel, S. (2015). Statewide administration of the CRAFFT screening tool: Highlighting the spectrum of substance use. Substance Use E' Misuse, 50, 1668-1677. doi:10.3109/108 26084.2015.1027930.

American Psychiatric Association [APA]. (2013). Diagnostic and statistical manual of mental disorders (fifth edition) (DSM-5). Washington, DC: American Psychiatric Publishing.

Araujo, M., Golpe, S., Braña, T., Varela, J. y Rial, A. (2018). Validación psicométrica del POSIT para el cribado del consumo de riesgo de alcohol y otras drogas en adolescentes. Adicciones, 30, 130-139. doi: 10.20882/adicciones.958.

Babor, T.F., De La Fuente, J.R., Saunders, J. y Grant, M. (1989). AUDIT: The Alcohol Use Disorders Identification Test. Guidelines for use in primary health care. Geneva, Switzerland: World Health Organization.

Bernard, M., Bolognini, M., Plancherel, B., Chinet, L., Laget, J., Stephan, P. y Halfon, O. (2005). French validity of two substance-use screening test among adolescents: A comparison of the CRAFFT and DEP-ADO. Journal of Substance Use, 10, 385-395. doi:10.1080/146598904123 31333050.

Bertini, M.C., Busaniche, J., Baquero, F., Eymann, A., Krauss, M., Paz, M. y Catsicaris, C. (2015). Adaptación transcultural y validación del test CRAFFT como prueba de pesquisa para consumo problemático, abuso y dependencia de alcohol y otras sustancias en un grupo de adolescentes argentinos. Archivos Argentinos de Pediatría, 113, 114-118. doi:10.5546/aap.2015.114.

Byrne, B. M. (2009). Structural equation modeling with AMOS: Basic concepts, applications, and programming (2nd ed.). London: Psychology Press.

Cadaveira, F. (2009). Alcohol y cerebro adolescente. Adicciones, 21, 9-14.

Calafat, A. y Juan, M. (2003). De la etiología a la prevención del uso y abuso de drogas recreativas. Adicciones, 15, 261-287.

Committee on Substance Abuse. (2011). Substance use screening, brief intervention, and referral to treatment for pediatricians. Pediatrics, 128, e1330-1340.

Cook, R.L., Chung, T., Kelly, T.M. y Clark, D.B. (2005). Alcohol screening in young persons attending a sexually transmitted disease clinic. Comparison of AUDIT, CRAFFT, and CAGE instruments. Journal of General Internal Medicine, 20, 1-6. doi:10.1111/j.1525-1497.2005.40052.x

Cortés, M.T., Espejo, B. y Giménez, J.A. (2007). Características que definen el fenómeno del botellón en universitarios y adolescentes. Adicciones, 19, 357-372.

Cote-Menendez, M., Uribe-Isaza, M.M. y Prieto-Suárez, E. (2013). Validación para Colombia de la escala CRAFFT para tamización de consumo de sustancias psicoactivas en adolescentes. Revista de Salud Pública, 15, 220-232.

Cummins, L.H., Chan, K.K., Burns, K.M., Blume, A.W., Larimer, M. y Marlatt, G.A. (2003). Validity of the CRAFFT in American-Indian and Alaska-Native adolescents: Screening for drug and alcohol risk. Journal of Studies on Alcohol, 64, 727-732. doi:10.15288/jsa.2003.64.727.

DeCamp, W., Gealt, R., Martin, S., O’Connell, D. y Visher, C. (2015). Binge drinking and other risk behaviors among college students. Newark, DE. Recuperado de https:// www.cdhs.udel.edu/content-sub-site/Documents/DDATA/2015 CRBS Report.pdf.

Dhalla, S., Zumbo, B.D. y Poole, G. (2011). A review of the psychometric properties of the CRAFFT instrument: 1999-2010. Current Drug Abuse Reviews, 4, 57-64. doi:10.2 174/1874473711104010057.

Dieppe, C., Stanhope, B. y Rakhra, K. (2009). Children who harm themselves: Development of a paediatric emergency department triage tool. Emergency Medicine Journal, 26, 418-420. doi:10.1136/emj.2008.062463.

ESPAD Group. (2016). ESPAD Report 2015: Results from the European School Survey Project on Alcohol and Other Drugs. 
Luxembourg. Recuperado de http://www.espad.org / sites/espad.org/files/ESPAD_report_2015.pdf.

Fontes, M.A., Bolla, K.I., Cunha, P.J., Almeida, P.P., Jungerman, F., Laranjeira, R.R., ... Lacerda, A.L. (2011). Cannabis use before age 15 and subsequent executive functioning. The British Journal of Psychiatry, 198, 442-447. doi:10.1192/ bjp.bp.110.077479.

Golpe, S., Isorna, M., Barreiro, C., Braña, T. y Rial, A. (2017). Consumo intensivo de alcohol en adolescentes: prevalencia, conductas de riesgo y variables asociadas. Adicciones, 29, 256-267. doi: 10.20882/adicciones.932.

Gryczynski, J., Kelly, S.M., Mitchell, S.G., Kirk, A., O’Grady, K.E. y Schwartz, R.P. (2015). Validation and performance of the Alcohol, Smoking and Substance Involvement Screening Test (ASSIST) among adolescent primary care patients. Addiction, 110, 240-247. doi:10.1111/ add. 12767.

Harris, S.K., Knight, J.R., Van Hook, S., Sherritt, L., Brooks, T.L., Kulig, J.W., ... Saitz, R. (2016). Adolescent substance use screening in primary care: Validity of computer self-administered versus clinician-administered screening. Substance Abuse, 37, 197-203. doi:10.1080/088970 77.2015.1014615.

Harris, S.K., Louis-Jacques, J. y Knight, J.R. (2014). Screening and brief intervention for alcohol and other abuse. Adolescent Medicine: State of the Art Reviews, 25, 126-156.

Kandemir, H., Aydemir, Ö., Ekinci, S., Selek, S., Kandemir, S.B. y Bayazit, H. (2015). Validity and reliability of the Turkish version of the CRAFFT Substance Abuse Screening Test among adolescents. Neuropsychiatric Disease and Treatment, 11, 1505-1509. doi:10.2147/NDT.S82232.

Karila, L., Legleye, S., Beck, F., Corruble, E., Falissard, B. y Reynaud, M. (2007). Validation d'un questionnaire de repérage de l'usage nocif d'alcool et de cannabis dans la population générale: le CRAFFT-ADOSPA. La Presse Médicale, 36, 582-590. doi:10.1016/j.lpm.2007.01.005.

Kelly, T.M., Donovan, J.E., Chung, T., Cook, R.L. y Delbridge, T.R. (2004). Alcohol use disorders among emergency department-treated older adolescents: A new brief screen (RUFT-Cut) using the AUDIT, CAGE, CRAFFT, and RAPS-QF. Alcoholism: Clinical E Experimental Research, 28, 746-753. doi:10.1097/01. ALC.0000125346.37075.85.

Kline, R.B. (2005). Principles and Practice of Structural Equation Modeling (2nd ed.). New York: The Guilford Press.

Knight, J.R., Sherritt, L., Harris, S.K., Gates, E.C. y Chang, G. (2003). Validity of brief alcohol screening test among adolescents: A comparison of the AUDIT, POSIT, CAGE, and CRAFFT. Alcoholism: Clinical E Experimental Research, 27, 67-73. doi:10.1111/ j.1530-0277.2003.tb02723.x.

Knight, J.R., Sherritt, L., Shrier, L.A., Harris, S.K. y Chang, G. (2002). Validity of the CRAFFT Substance Abuse Screening Test among adolescent clinical patients. Ar- chives of Pediatrics $\mathcal{E}$ Adolescent Medicine, 156, 607-614. doi:10.1001/archpedi.156.6.607.

Knight, J.R., Shrier, L.A., Bravender, T.D., Farrell, M., Vander Bilt, J. y Shaffer, H.J. (1999). A new brief screen for adolescent substance abuse. Archives of Pediatrics $\mathcal{E}^{\circ}$ Adolescent Medicine, 153, 591-596. doi:10.1001/archpedi.153.6.591.

Kuder, G.F. y Richardson, M.W. (1937). The theory of the estimation of test reliability. Psychometrika, 2, 151-160.

Latimer, W., Winters, K.C. y Stinchfield, R.D. (1997). Screening for drug abuse among adolescents in clinical and correctional settings using the Problem Oriented Screening Instrument for Teenagers. The American Journal of Drug and Alcohol Abuse, 23, 79-98. doi:10.3109/00952999709001689.

López-Caneda, E., Mota, N., Crego, A., Velasquez, T., Corral, M., Rodríguez, S. y Cadaveira, F. (2014). Anomalías neurocognitivas asociadas al consumo intensivo de alcohol (binge drinking) en jóvenes y adolescentes: una revisión. Adicciones, 26, 334-359.

Mitchell, S.G., Kelly, S.M., Gryczynski, J., Myers, C.P., O'Grady, K.E., Kirk, A S. y Schwartz, R P. (2014). The CRAFFT cut-points and DSM- 5 criteria for alcohol and other drugs: A reevaluation and reexamination. Sustance Abu se, 35, 376-380. doi:10.1080/08897077.2014.936992.

Moure, L., Caamaño, F., Doallo, S., Juan, P., Corral, M., Rodríguez, S. y Cadaveira, F. (2014). Heavy drinking and alcohol related injuries in college students. Gaceta Sanitaria, 28, 376-380. doi:10.1016/j.gaceta.2014.02.017.

National Institute on Alcohol Abuse and Alcoholism [NIAAA]. (2011). Alcohol screening and brief intervention for youth: A practitioner's guide. Rockville, MD. Recuperado de https://pubs.niaaa.nih.gov/OrderForm/EncForm/Youth_Guide_Order_Form

Parada, M., Corral, M., Caamaño, F., Mota, N., Crego, A., Rodríguez, S. y Cadaveira, F. (2011). Definición del concepto de consumo intensivo de alcohol adolescente (binge drinking). Adicciones, 23, 53-63.

Pereira, B.A., Schram, P.F. y Azevedo, R.C. (2016). Evaluation of the Brazilian version of the CRAFFT/CESARE scale for screening drug use by adolescents. Ciência Ẽ Saúde Coletiva, 21, 91-99. doi:10.1590/141381232015211.05192015.

Pérez, A. y Scoppetta, O. (2011). El CRAFFT/CARLOS como instrumento para la identificación temprana de consumo de alcohol y otras SPA: una adaptación al español. Revista Colombiana de Psicología, 20, 265-274.

Pilowsky, J. y Wu, L.T. (2013). Screening instruments for substance use and brief interventions targeting adolescents in primary care: A literature review. Addictive Behaviors, 38, 2146-2153. doi:10.1016/j.addbeh.2013.01.015.

Plan Nacional sobre Drogas. (2016). Encuesta sobre el uso de drogas en enseñanzas secundarias en España (ESTUDES) 2014-2015. Madrid, España: Delegación del Gobierno 
para el Plan Nacional sobre Drogas, Ministerio de Sanidad, Servicios Sociales e Igualdad.

Rahdert, E.R. (1991). The Adolescent Assessment/Referral System. Rockville, MD: National Institute on Drug Abuse.

Sánchez, I., Moreno, C., Rivera, F. y Ramos, P. (2015). Tendencias en el consumo de alcohol en los adolescentes escolarizados españoles a lo largo de la primera década del siglo XXI. Gaceta Sanitaria, 29, 184-189. doi:10.1016/j.gaceta.2015.01.004.

Skogen, J.C., BØe, T., Knudsen, A.K. y Hysing, M. (2013). Psychometric properties and concurrent validity of the CRAFFT among Norwegian adolescents. Ung@hordaland, a population-based study. Addictive Behaviors, 38, 2500-2505. doi:10.1016/ j.addbeh.2013.05.002.

Subramaniam, M., Cheok, C., Verma, S., Wong, J. y Chong, S. A. (2010). Validity of a brief screening instrument-CRAFFT in a multiethnic Asian population. Addictive Behaviors, 35, 1102-1104. doi:10.1016/j.addbeh.2010.08.004.

Van Weelden, M., Lourenço, B., Viola, G.R., Aikawa, N.E., Queiroz, L.B. y Silva, C.A. (2016). Substance use and sexual function in juvenile idiopathic arthritis. Revista Brasileira de Reumatología, 56, 323-329. doi:10.1016/j. rbre.2016.02.007.

Wartberg, L., Kriston, L., Diestelkamp, S., Arnaud, N. y Thomasius, R. (2016). Psychometric properties of the German version of the CRAFFT. Addictive Behaviors, 59, 42-47. doi:10.1016/j.addbeh.2016.03.020.

Winters, K C. y Henly, G. (1993). Adolescent Diagnostic Interview (ADI). Los Ángeles, CA: Western Psychological Services.

Winters, K.C., Stinchfield, R.D., Henly, G.A. y Schwartz, R.H. (1990). Validity of adolescent self-report of alcohol and other drug involvement. International Journal of the Addictions, 25, 1379-1395. doi:10.3109/10826089009068469. 